\title{
Poor Man's Guide to Celestial Mechanics
}

by

\author{
HANNES ALFVÉN \\ Royal Institute of Technology, \\ Stockholm
}

THE principal problem in celestial mechanics has been to calculate the exact positions of celestial bodies at any instant, given a number of very accurate observations. In some cases, however, for example, in connexion with problems concerned with the evolution of the solar system, we are more interested in the long term changes in the orbits, the interaction between a body and the plasma out of which it has condensed, and the mutual interactions between a large number of planetesimals. In such cases the usual methods are not very convenient. They can be replaced by the following method which is related to ordinary methods just as in the theory of electric particles in a magnetic field the guiding centre method is related to the Störmer method.

Suppose that in a cylindrical coordinate system $(r, \varphi, z)$ a particle moves with velocity $\left(v_{r}, v_{\varphi}, v_{z}\right)$ under the action of gravitation $\left(f_{r}, 0, f_{z}\right)$ from a central body, which is an ellipsoid, flattened due to rotation around the $z$ axis.

If the particle moves in the equatorial plane, the angular momentum is constant

$$
C=r v_{0}
$$

The centrifugal force is

$$
f_{c}=\frac{v_{\varphi}^{2}}{r}=\frac{C^{2}}{r^{3}}
$$

If the particle moves in a circle with radius $r_{o}$, we have $f_{c}=f_{r_{-}}{ }_{-}$pnd the angular velocity is

$$
\omega_{\varkappa}=\left(-f_{r} / r_{o}\right)^{1 / 2}
$$

We disturb the motion by displacing the body a small distance $\Delta r$ in the radial direction. It is acted on by the force

$$
\Delta f_{r}=\Delta\left(f_{e}+f_{r}\right)=\left(-\frac{3 C^{2}}{r^{4}}+\frac{\partial f_{r}}{\partial r}\right) \Delta r
$$

which means that it can oscillate in the radial direction with a frequency

$$
\omega_{r}=\left(\frac{3 C^{2}}{r^{4}}-\frac{\partial f_{r}}{\partial r}\right)^{1 / 2}=\left(-\frac{3 f_{r}}{r}-\frac{\partial f_{r}}{\partial r}\right)^{1 / 2}
$$

If the body is displaced $\Delta z$ in the $z$ direction, it is acted on by the force $\Delta f_{z}$ which because of $\operatorname{div} f=0$ is given by

$$
\Delta f_{z}=-\frac{1}{r} \frac{\partial}{\partial r}\left(r f_{r}\right) \Delta z=-\left(\frac{f_{r}}{r}+\frac{\partial f_{r}}{\partial r}\right) \Delta z
$$

And so the frequency for axial oscillations is

$$
\omega_{2}=\left(\frac{f_{r}}{r}+\frac{\partial f_{r}}{\partial r}\right)^{1 / 2}
$$

From equations (3), (5) and (7) we find

$$
\omega_{r}^{2}+\omega_{2}^{2}=2 \omega_{x}^{2}
$$

If the central force is a coulomb field (that is, the central body is a sphere), we have

$$
\frac{\partial f_{r}}{\partial r}=\frac{f_{r}}{r}
$$

and our problem is degenerate in the sense that

$$
\omega_{r}=\omega_{z}=\omega_{\varkappa}
$$

The relation $\omega_{r}=\omega_{\varkappa}$ means that in the equatorial plane the particle moves in an eccentric orbit (ellipse). On this motion may be superimposed axial oscillations of the same frequency, meaning that the plane of the ellipse may have an inclination with regard to the equatorial plane (which in this case is arbitrary).

If the central body rotates so that it becomes an ellipsoid, and its axis coincides with our $z$ axis, the central force in the equatorial plane has a fourth degree term (and possibly also higher terms). As in this case

$$
\left|\frac{\partial f_{r}}{\partial r}\right|>\frac{f_{r}}{r}
$$

we have

$$
\omega_{z}>\omega_{x}>\omega_{r}
$$

This means that the perihelion moves with velocity

$$
\omega_{\pi}=\omega_{x}-\omega_{r}>0
$$

and the nodes move with velocity

$$
\omega_{\theta}=\omega_{x}-\omega_{z}<0
$$

Further from equations (8), (13) and (14)

$$
\omega_{\pi}+\omega_{\theta}=\frac{\omega_{\pi}^{2}+\omega_{\theta}^{2}}{\left.2 \omega_{x}\right]}
$$

Because the right hand term is very small we find to a first approximation

$$
\omega_{\pi}=-\omega_{\theta}
$$

which is a well known result in celestial mechanics.

Introducing equation (16) into equation (15) gives as a second approximation

$$
\Delta \omega=\omega_{\pi}+\omega_{\theta}=\frac{\omega_{\pi}^{2}}{\omega_{x}}
$$

Brouwer ${ }^{1}$ has treated the case when a satellite moves so close to a rotating central body that the fourth degree term of the equatorial bulge becomes important, and applied the result to the motion of Amalthea (Jupiter V), for which we know from observations that $\omega_{x}=722^{\circ}$ day $^{-1}=722.365^{\circ}$ year ${ }^{-1} ; \omega_{\pi}=917^{\circ} .4$ year $^{-1}$, and $\omega_{\theta}=$ $-915^{\circ} .7$ year $^{-1}$. From an analysis by the usual methods of celestial mechanies, Brouwer finds $\Delta \omega=3^{\circ} \cdot 2$ year $^{-1}$, which is in reasonable agreement with the observational value $2^{\circ} .7$. Our formula (5) gives

$$
\Delta \omega=\frac{917^{2}}{722 \cdot 365}=3^{\circ} .16 \text { year }^{-1}
$$

And so for this case the simple approximate method seems to be in good agreement with the elaborate treatment by the usual methods.

1 Brouwer, D., Astron. J., 51, 223 (1945) 